\title{
Multiresolution mesh segmentation based on surface roughness and wavelet analysis
}

\author{
Céline Roudet $^{a}$, Florent Dupont ${ }^{a}$ and Atilla Baskurt ${ }^{b}$ \\ ${ }^{a}$ Laboratoire LIRIS, UMR 5205 CNRS, Université Lyon 1, Villeurbanne, F-69622, France. \\ ${ }^{b}$ Laboratoire LIRIS, UMR 5205 CNRS, INSA de Lyon, Villeurbanne, F-69621, France.
}

\begin{abstract}
During the last decades, the three-dimensional objects have begun to compete with traditional multimedia (images, sounds and videos) and have been used by more and more applications. The common model used to represent them is a surfacic mesh due to its intrinsic simplicity and efficacity. In this paper, we present a new algorithm for the segmentation of semi-regular triangle meshes, via multiresolution analysis. Our method uses several measures which reflect the roughness of the surface for all meshes resulting from the decomposition of the initial model into different fine-to-coarse multiresolution meshes. The geometric data decomposition is based on the lifting scheme. Using that formulation, we have compared various interpolant prediction operators, associated or not with an update step. For each resolution level, the resulting approximation mesh is then partitioned into classes having almost constant roughness thanks to a clustering algorithm. Resulting classes gather regions having the same visual appearance in term of roughness. The last step consists in decomposing the mesh into connex groups of triangles using region growing ang merging algorithms. These connex surface patches are of particular interest for adaptive mesh compression, visualisation, indexation or watermarking.
\end{abstract}

Keywords: Mesh segmentation, classification, multi-resolution analysis, geometric wavelet, lifting scheme, region growing, region merging.

\section{INTRODUCTION}

The complexity of the 3D models, used in computer graphics, have recently increased due to the last progress in sampling techniques. These objects are consequently represented numerically with more and more precision and details in order to answer realism waitings. Triangle mesh is the most common representation for these objects because it's a well adapted model for many applications and for the rendering process. This representation include geometry and topology information which could be expensive for computation, storage, transmission, and display tasks, even if the material involved is more and more competitive. Consequently multiresolution (MR) techniques have emerged, in order to represent data with multiple Level Of Detail (LOD) for progressive transmission and visualisation purposes, where a coarse approximation can subsequently be further improved depending on the user ressources and waitings. In order to adapt to the heterogeneity of the material involved, MR analysis is employed to produce such LODs thanks to wavelet transform. This latter transform is mainly used by many authors for progressive compression and visualisation, but also for denoising, filtering or surface editing purposes.

The goal of this work is to benefit from MR analysis to produce a serie of segmentations for all resolution levels. Segmentation is used in many computer graphics applications, like mesh compression or simplification which can be simplified on surface patches. For texture mapping, it allows a simplification of the parameterization for high genus meshes. Metamorphosis or animation can also benefit from mesh decomposition in order to establish a correspondance between each part of the objects. Most of existing segmentation algorithms are based on the curvature or planarity information to distinguish the relevant parts of the object. They consist in decomposing the original model in regions having the same characteristics in term of curvature. This treatment is easier when dealing with CAO objects which contain sharp edges and corners that separate generally smooth regions.

Corresponding author currently at Université Lyon 1, Bâtiment NAUTIBUS, 8 boulevard Niels Bohr, 69622 Villeurbanne Cedex - France. Email: croudet@liris.cnrs.fr, Telephone: +33 (0)4 262344 64, Fax: +33 (0)4 72431536. 
In this paper we propose a new mesh segmentation algorithm based on roughness information computed locally on the surface. For some kind of objects, like the Venus model presented in Fig. 4, the obtained regions correspond to semantic parts, because people have a tendancy to distinguish parts according to their frequency. Considering that the roughness of the surface is intrinsically linked to multiresolution, our framework will be able to better take into account this kind of measure.

Having a different segmentation for each resolution level, based on roughness information could be interesting for indexation where each one contains various levels of frequencies while keeping the global form of the initial mesh. Watermarking and compression can also benefit from this framework in order to apply different marks or subdivision schemes according to the visual aspect of the surface. Finally, it can increase the adaptiveness for visualisation and navigation in large scenes purposes, where parts of objects or scenes could be more refined than others, taking into account the user's point of view and interest.

In the next paragraph, we review briefly recent papers dealing with multiresolution analysis and segmentation of triangular meshes. In sect. 3, we detail our segmentation approach based on the coefficients produced by a wavelet decomposition. Sect. 4 presents the results we have obtained. We finish by a discussion and ideas for future work.

\section{RELATED WORK}

One of our principal objectives is to develop a new adaptive and progressive mesh compression algorithm, aiming at applying various wavelet decompositions and quantizations dependending on the roughness of the surface. Consequently a surface partition is first needed. Within this framework, we have considered the distribution of the wavelet coefficients norm and polar angle, obtained by a global multiresolution (MR) analysis and which reflects the roughness of the surface. Before detailing our algorithm, we present the existing work done in MR analysis of semi-regular meshes and in mesh segmentation.

\subsection{State of the art in multiresolution analysis of semi-regular meshes}

MR analysis of meshes with arbitrary topology was introduced by Lounsbery ${ }^{1}$ who have used a canonical subdivision of the facets and a geometric wavelet transform to obtain multiple resolution levels. He showed that a subdivision scheme can serve as a scaling functions basis in order to extend the wavelet theory for irregular sampled signals like meshes. Most of the algorithms dealing with MR analysis apply it to semi-regular meshes obtained by remeshing techniques and favourable with the extension of the MR analysis scheme proposed by Lounsbery. ${ }^{1}$ In this way, meshes can be considered as functions via the produced parameterization. These papers diverge by the method used to construct a good approximation of the initial surface with subdivision connectivity, which can be done while refining a coarse mesh, obtained directly from the initial object ${ }^{2,3}$ or by progressive decimations. ${ }^{4,5}$

The first remeshing method ${ }^{2}$ proposed within this framework use a partition of the original mesh into Voronoï tiles, computed using the geodesic distance. The coarse model is obtained using the dual construction : the Delaunay triangulation. This latter is then refined by subdivision steps and additional displacements to obtain a semi-regular approximation of the original form. The details missed by the subdivision operation are determined at each resolution level thanks to a local parameterization, based on harmonic maps. In the same way, Gioia ${ }^{3}$ have also used a parameterization based on harmonic maps to produce a semi-regular mesh from a coarse one obtained by a partitioning process. The principal difference between these methods is that the latter take more into account the geometric and visual properties of the initial surface during the construction of the parameterization and the coarsest approximation. Gioia experimentally obtain on average twice less wavelet coefficients than with previous method, for natural and CAD objects, considering the fact that we are dealing with geometric shapes and not just functions.

The other concept for building a semi-regular mesh was introduced by Lee et al. ${ }^{4}$ who have constructed the coarse mesh thanks to a decimated process based on local simplifications of the initial model. Vertex removals are used to progressively build a parameterization of the original object in a hierarchy of meshes. At each step, the vertices choosen to be removed are those that withdraw the fewest geometrical and topological information. The parameterization consists in expressing the decimated vertices as barycentric coordinates of the resulting 
ones. The Loop subdivision ${ }^{6}$ is then used to produce the semi-regular mesh from the coarsest one. The details added at each resolution are represented by $3 \mathrm{D}$ vectors. Another famous algorithm based on the same concept ${ }^{5}$ benefits from other type of parameterization and a non lifted Butterfly subdivision scheme ${ }^{7}$ to concentrate the high-frequency information along the surface normal which could be expressed by a scalar.

The application of the MR analysis on the semi-regular meshes resulting from these latter algorithms and mainly used for progressive compression purposes, can be based on various subdivision schemes. Most of the existing methods ${ }^{1,3,8,9}$ benefit from interpolating subdivision schemes for the low-resolution versions to be good approximations of the original object (in a least-squares sense). In other words, to provide numerical stability of the fitting operation. But other authors ${ }^{10,11}$ have recently proposed a wavelet construction for the Loop subdivision, based on the lifting scheme. Contrary to Khodakovsky filters, ${ }^{12}$ constructed previously, the wavelet analysis and synthesis are obtained in linear time, providing relatively stable schemes.

All of these previously described algorithms apply a global wavelet decomposition, using the same schemes on the entire surface of the mesh. Our objective is to apply different analysis and synthesis schemes for regions that don't exhibit the same roughness degree, in order to obtain better compression results. For that purpose, we first need a mesh decomposition.

\subsection{State of the art in mesh segmentation}

Image segmentation have received considerable attention since last years, because there is a large number of higher-level image processing problems that can benefit from this decomposition as a preprocessing step. In the same way, lots of work have been done in segmentation of 3D data, mainly based on range images or point clouds representations. But more recently, authors have proposed algorithms for mesh segmentation which can be decomposed in two groups, depending whether they are interpreted in a purely geometric sense or in a more semantic-oriented manner. In the latter case, the object is decomposed in parts corresponding to relevant aspects of the surface of the shape (sub-meshes). This principle can be used for applications seeking the meaningful components of the objects as collision detection, skeletonization, metamorphosis, animation or modeling by parts, which are far from our expectations.

Methods that belong to the first category partition the object in surface patches having common characteristics (planarity, constant curvature, ...). Some of them intend to approximate the object by planar faces and to minimize approximation error between this set of elements and the original surface. ${ }^{13,14}$ These approximation techniques are particularly useful for mesh simplification and radiosity. Other approaches ${ }^{15,16}$ use the discrete curvature computed in each vertex with a watershed algorithm adapted from those employed in image segmentation. More recently, Razdan et al. ${ }^{17}$ have proposed a hybrid approach, which combine the watershed algorithm with a sharp edge extraction. But theses methods tend to extract only regions surrounded by high curvatures and don't handle correctly the boundaries between the patches, which are either fuzzy or jagged. The method developped by Lavoué et al. ${ }^{18}$ overcome these drawbacks using a K-Means classification algorithm ${ }^{19}$ instead of the watershed, in order to more precisely detect curvature transitions, particularly on CAD objects.

We propose to adapt this latter algorithm to natural objects using the wavelet coefficients as roughness measure. They represent the high-frequencies lost during the coarsification of the initial model and reflect the roughness of the surface, as less details are needed in smooth regions than in textured or noisy ones.

\section{PROPOSED METHOD}

In this section, we first detail the theory involved to produce the wavelet coefficients. We present then the various $\mathrm{MR}$ analysis schemes we have compared to obtain the best mesh decomposition. We finally describe our adaptation of the mesh segmentation algorithm introduced by Lavoué et al. ${ }^{18}$

\subsection{Wavelet theory and lifting scheme}

The MR analysis produces a reversible decomposition of a mesh into a serie of approximation meshes and a sequence of wavelet coefficients. Classical MR analysis methods such as wavelet transform are based on the filter 


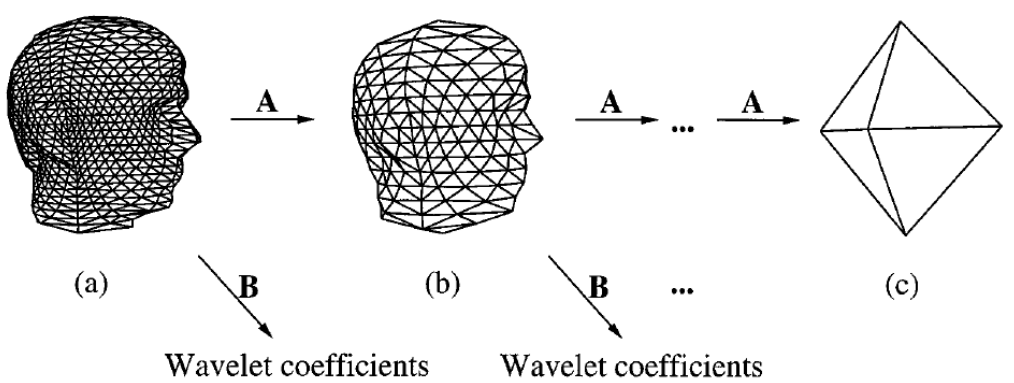

Figure 1. Decomposition of a polyhedral surface.

bank theory where lowpass and highpass filters are applied in order to obtain respectively approximations and details. For a discrete signal $s(t)$, its Wavelet Transform is explained by this formulation :

$$
W T[s(t)](m, n)=<\psi_{n}^{m}, s>=a_{0}^{-\frac{m}{2}} \int_{-\infty}^{+\infty} s(t) \psi^{*}\left(a_{0}^{-m} t-n b_{0}\right) d t .
$$

where $m, n \in \mathbb{Z}, a_{0}>1, b_{0}>0$ and $\psi^{*}$ corresponds to the complex conjugation of $\psi$.

The choice of $a_{0}$ and $b_{0}$ produces different tilings of the time-frequency plane. The most used technique is the dyadic analysis, introduced by Mallat ${ }^{20}$ in 1989 to link the wavelets and the filter bank theories, where $a_{0}=2$ and $b_{0}=1$. This MR analysis formulation, defined for finite energy functions, is formally described for a serie of imbricated subspaces $\left(V^{m}\right)_{m \in \mathbb{Z}} \subset L^{2}(\mathbb{R})$ in which an orthonormal basis of scaling functions is determined. This family of functions $\left\{\varphi_{n}^{m} ; n \in \mathbb{Z}\right\}$ is defined by dilations and translations of a mother scaling function $\varphi(t)$ and expressed by the following formula : $\varphi_{n}^{m}(t)=2^{-m / 2} \varphi\left(2^{m} t-n\right)$. The orthogonal projection of $s(t)$ into the scaling functions family forms an approximation of the signal, which is interpreted as a lowpass filter followed by a uniform subsampling. To recover the high frequencies losts during the preceding step, we have to consider the $W^{m}$ spaces, which are the orthonormal complements of the $V^{m}$ space in the $V^{m-1}$ one. The wavelets, defined by $\psi_{n}^{m}(t)=2^{-m / 2} \psi\left(2^{m} t-n\right)$, form an orthonormal basis of the $W^{m}$ spaces and are used as highpass filtering in order to collect the missed details.

This formulation can be applied to meshes, using subdivision as lowpass filtering, as defined by Lounsbery and shown in Fig. 1. But in practice it's interesting to benefit from wavelets having at least one vanishing moment, especially for compression purposes in order to benefit from a decrease of the wavelet coefficients through the resolution levels. The orthogonality of the wavelets with the scaling functions is also sought to obtain the best approximations in a least square sense which is important for visualisation and to improve coding performances. This orthogonalisation means that $\left\langle\varphi_{i}^{j}, \psi_{k}^{j}\right\rangle=0$ for each pair $(i, k) \in \mathbb{Z}^{2},\langle f, g\rangle$ being defined as the inner product between the functions $f$ and $g$. The inner product on a triangular mesh $M$, was first defined by Lounsbery and is computed with the following formula:

$$
<f, g>=\sum_{\tau \in \Delta(M)} \frac{1}{\operatorname{Area}(\tau)} \int_{s \in \tau} f(s) \cdot g(s) \cdot d s
$$

where $\Delta(M)$ denotes the set of triangular faces of $M$ and $d s$ is the usual Euclidean area form for the triangles $\tau$ in $\mathbb{R}^{3}$.

But it's not always possible to construct analysis tools having such properties from the filter bank theory. Consequently most of the methods benefit from the lifting scheme formulation, introduced by Sweldens ${ }^{21}$ which moreover reduce computational costs and memory allocation by first splitting the signal into even and odd components using lazy wavelets and represented by $S$ in Fig. 2. The following lifting steps of Fig. 2 produce a modification of this biorthogonal wavelet basis in order to add the desired properties. These operations are 


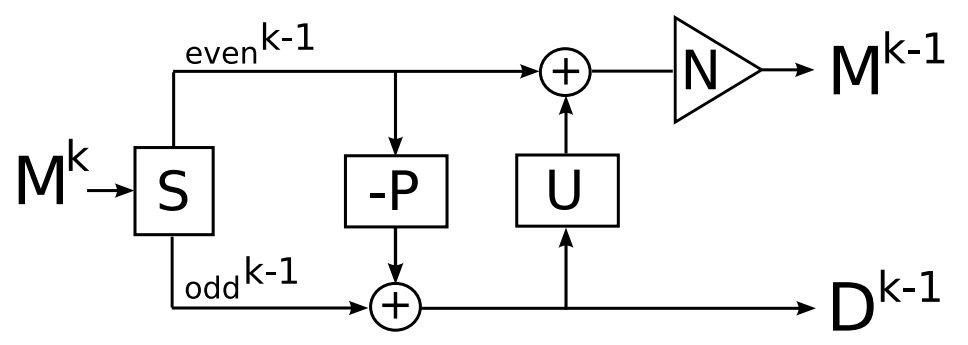

Figure 2. Principle of the lifting scheme for the decomposition of a fine mesh $\left(M^{k}\right)$ into a coarser one $\left(M^{k-1}\right)$ and details $\left(D^{k-1}\right)$.

equivalent to a factorization of the pair of complex filters which can be simplified by the lifting formulation. Hence, the MR analysis is simply obtained with finite filters by an inversion of the order and signs of the synthesis lifting matrices.

The prediction operator $(P)$ is used to predict odd components from even ones, in order to obtain smaller coefficients by better approaching the higher level mesh than with a canonical quadrisection. The update operator $(U)$ is used to preserve the mean value of the signal and corresponds to the addition of the previously cited properties, like orthogonalisation of the scaling functions $\varphi$ with the wavelets $\psi$.

This latter property is applied to minimize the norm of the orthogonal projection of the $\psi_{i}^{j}$ on the $V^{j}$ spaces, $\forall i, j \in \mathbb{Z}$, with respect to the inner product. Hence the wavelets are expressed by :

$$
\psi_{i}^{j}=\varphi_{i}^{j+1}+\sum_{k \in \nabla_{j}} \alpha_{i k} \varphi_{k}^{j}
$$

with $\nabla_{j}$ defined as the set of vertices of $M^{j}$.

The coefficients $\alpha_{i k}$ of this latter equation are the solution of a linear system which depends on the desired properties. In practice, the orthogonalisation is relaxed in order to obtain finite filters. For compression purposes, the decomposition ends up with a normalisation step $(N)$, which is used to adjust the scale between odd and even coefficients so as to decompose the surface in a normalised basis.

\subsection{Construction of various lifting schemes}

In the framework of our segmentation perspective, based on roughness, we have compared different interpolant prediction schemes associated or not with an update step. The following interpolant schemes were choosen because they produce a more stable decomposition than with approximant ones :

- the midpoint scheme which corresponds to a canonical quadrisection of the facets (addition of the new vertices in the middle of each edge) ;

- the Butterfly scheme introduced by Dyn et al., ${ }^{7}$ which produces a $C^{1}$ limit surface for meshes having a regular topology. Moreover it's the interpolant scheme with the smallest stencil which allows to reduce computational costs ;

- the extension of the Butterfly scheme proposed by Zorin et al. ${ }^{22}$ who have introduced new masks for extraordinary vertices while preserving the simplicity and the behaviour of the original scheme. The new weights are computed with a discrete Fourier transform and a principal component analysis. Unlike the other interpolant schemes, it produces comparable results than with approximant stencils ;

- a Butterfly extension we have proposed with smaller stencils than those used by Zorin et al. for extraordinary vertices. 
We also have compared two formulations of the lifting scheme where the prediction and the update steps can be reversed. For this latter step, we only have employed a 0-ring stencil, taking into account Certain et al. ${ }^{23}$ assumptions. They indeed have compared experimentally the performance of the lazy, 0-, 1- and 2-disk wavelets to know how quickly the geometric error decreases as a function of the number of wavelet coefficients or polygons in the model. The experimental results have shown that the lazy wavelets perform slightly worse than the $k$-disk ones, but there is no significant difference between the various values of $k$. Moreover, the wavelets with smaller supports are likely to be more stable numerically, which is important as the number of levels increases.

Finally, the MR analysis framework was applied on semi-regular meshes obtained by the two most famous algorithms which define a parameterization on a hierarchy of meshes. The first is called MAPS ${ }^{4}$ and the other produces Normal meshes. ${ }^{5}$

\subsection{Wavelet decomposition for segmentation purposes}

The mesh segmentation in surface patches having a quasi-constant roughness has been computed using an adaptation of the algorithm proposed by Lavoué et al. ${ }^{18}$ (originally conceived for CAD objects). This latter exploits the principal curvature values, computed in each vertex using the estimation of the curvature tensors defined by Cohen-Steiner and Morvan. ${ }^{24}$

More precisely, they first have used a pre-processing step to detect sharp edges, particularly found in optimized triangulated CAD objects, where curvature is theoretically not defined. As we intend to treat natural objects and not specifically CAD ones, we don't have considered this sharp features detection.

Several authors have proposed different algorithms for estimating the curvature tensors, ${ }^{24-26}$ but the one introduced by Cohen-Steiner and Morvan, ${ }^{24}$ based on the Normal Cycle, has given better results even for CAD objects. Once these tensors have been estimated, their eigenvalues represent the principal curvature values $(\mathrm{kmin}$ and $k \max$ ) and have been used in Lavoué et al. classification algorithm. In this paper, we propose to replace this curvature information by our previously detailed roughness measure, to obtain a different segmentation for natural objects.

\subsubsection{Wavelet coefficients used to reflect surface roughness}

The contribution of this work is the use of the wavelet coefficients norm and polar angle (the angle between each coefficient and its corresponding surface normal vector), obtained by the various schemes explained in sect. 3.2, to differentiate mesh regions in term of roughness. We have considered two ranges for the polar angle measure. The first lies between 0 and $\pi / 2$ and doesn't differentiate vectors with opposite directions from those having the same, unlike the second which lies between 0 and $\pi$. We obtain a hierarchy of mesh segmentations having less and less high-frequencies, in which the wavelet coefficients are linked to edges.

The histograms presented at the first row of Fig. 3 show the non-uniform distribution of these measures for all the coefficients linked to the first decomposition level edges and obtained with the midpoint analysis. In order to compare these quantities with two other roughness measures linked to the vertices and to make the adaptation of the Lavoué et al. algorithm possible, we have computed for each vertex the mean of the values associated with its incident edges. The corresponding normalized distributions can be seen at the second row of Fig. 3 .

The first roughness measure we have used for comparison was defined by Lavoué et al. ${ }^{27}$ who have considered a local sphere centered in each vertex in which they have computed the standard curvature deviation $\sigma_{x}$. They have employed it to measure the visual similarity between 3D meshes. The second algorithm used to reflect roughness compute the normal difference between the model and a smoothed version obtained using a Laplacian smoothing. These quantities are shown for the Venus and rabbit models at the first row of Fig. 4 and 5 .

\subsubsection{Classification algorithm based on roughness}

The classification algorithm of Lavoué et al. ${ }^{18}$ is then used to create $K$ groups of vertices that could be userdefined. The vertices are classified according to our roughness values, associated with the Euclidean distance in the roughness space. After several iterations, starting with $K$ randomly determined centroïds, each vertex is then associated to a cluster $C_{i}$ and a roughness value $r_{i}$. A cluster regularization (merging of small or similar clusters) 


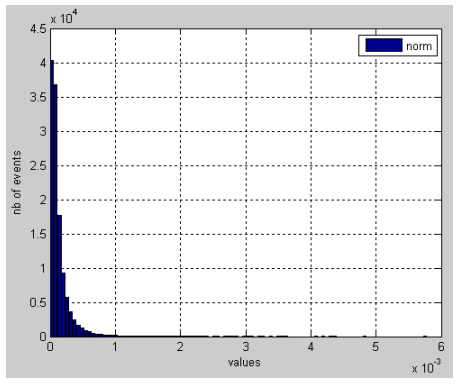

(a) Distribution of the wavelet coefficients norm (linked to edges) on the first
decomposition level ( 81920 faces)

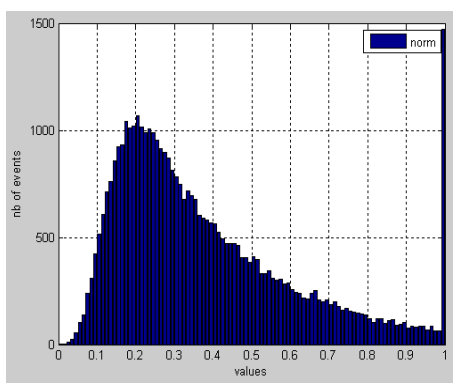

(d) Distribution of the wavelet coefficients

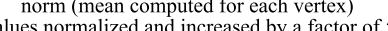

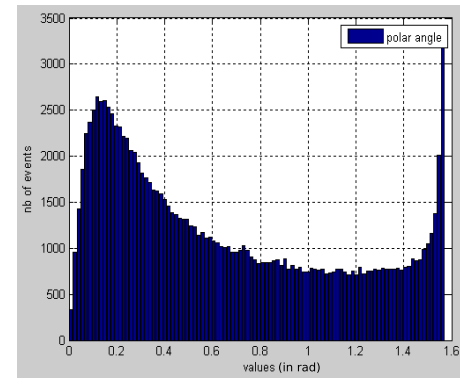

(b) Distribution of the wavelet coefficients polar angle (linked to edges and ranging between $0 \& 90^{\circ}$ )

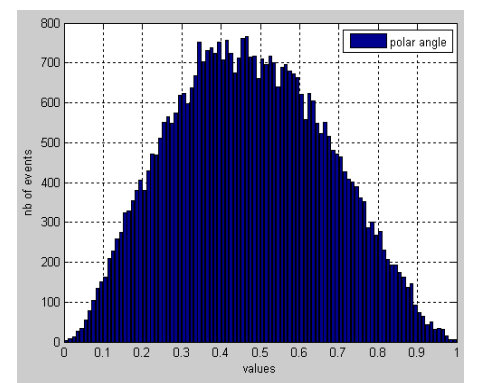

(e) Distribution of the wavelet coefficients polar angle (for values ranging between $0 \& 90^{\circ}$ )

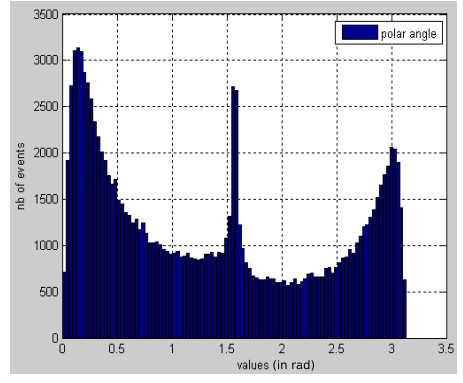

(c) Distribution of the wavelet coefficients polar angle (linked to edges ang ranging between $\left.0 \& 180^{\circ}\right)$

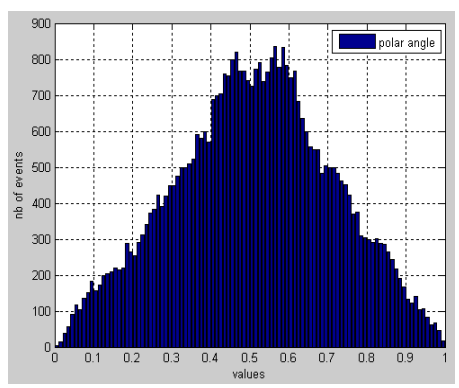

(f) Distribution of the wavelet coefficients polar angle (for values ranging between $0 \& 180^{\circ}$ )

Figure 3. Distribution of the wavelet coefficients norm and polar angle for the first resolution level of the Venus model (midpoint analysis). (a-c) Distribution obtained taking into account all the coefficients, linked to the model edges. (d-f) Normalized distribution of the mean values computed for each vertex from its incident edges. The wavelet coefficients norm have been increased by a factor in order to stretch the distribution originally concentrated near the origin

is finally added. For our purposes, two clusters are sufficient in order to separate only smooth regions from rough ones. Considering more clusters leads to a too detailed classification which produces over-segmentation.

The next step consists in constructing quasi-similar roughness connex regions composed of triangles from the two groups of vertices obtained in the roughness space.

\subsubsection{Region growing and merging algorithms}

We have used the region growing and labeling algorithm of Lavoué et al. ${ }^{18}$ in order to transmit the roughness information from vertices to triangles, starting from seed triangles having their three vertices on the same cluster. This treatment is then followed by a region merging algorithm which mainly aims at reducing the oversegmentation resulting from the growing step. This latter operation consists in constructing a region adjacency graph, where the nodes represent the regions produced by the preceding step and the edges an adjacency relation between two of them. A reduction of the graph is then processed where the smallest edge is eliminated at each iteration, resulting in a merging of the two corresponding parts. The graph reduction stops when the region number reaches a queried number or when the weight of the smallest edge is larger than a given threshold. This reduction is based on the similarity distance $D_{i j}$ which gather curvature similarity, size and common perimeter of the two regions. We are actually working to enrich $D_{i j}$ with a roughness similarity distance in order to obtain better results. The reader can refer to the corresponding paper ${ }^{18}$ for a more detailed presentation of these algorithms.

Thanks to this framework, we are now able to apply various subdivision schemes and wavelet coefficients quantizations, each one of them adapted to the surface roughness. 


\section{EXPERIMENTAL RESULTS}

The results shown have been computed with an application implemented in $\mathrm{C}++$ that uses the Computational Geometry Algorithm Library (CGAL) ${ }^{28}$ Our classification and segmentation methods, based on roughness measures, were tested on the semi-regular objects obtained thanks to the two remeshing algorithms considered (provided by Caltech). Examples are given for the most revealing objects in term of roughness : the Venus and the rabbit models. Fig. 4 and 5 present the various steps of our algorithm for these models.

\subsection{Comparison of wavelet decomposition}

The distribution of the wavelet coefficients norm, presented at the second row of Fig. 4 and on the model (e) of Fig. 5 is comparable with those obtained considering the two other roughness measures, detailed in sect. 3.3.1 and visible at the first row of Fig. 4 and 5 . The wavelet coefficients norm have been increased by a factor to stretch the distribution originally concentrated near the origin (as we can see on the first histogram of Fig. 3), allowing a better classification. We can see that these measures reflect the different aspects of the mesh surface, even for the Venus second decomposition level which has approximately $4^{2}$ times less vertices than the original object.

The study of the obtained results have shown that the inversion of the prediction and update steps does not have a meaningful signification. In the same way, the update operation doesn't modify significantly the results, so we don't have presented it in our figures.

While observing the various distributions of the coefficients norm, presented at the second row of Fig. 4, we can notice that the results obtained with the midpoint and Butterfly analysis on the Normal mesh are comparable. Nevertheless the Butterfly scheme allow a better differentiation between smooth and rough parts. This tendancy to obtain smaller wavelet coefficients in smooth regions with the Butterfly scheme can be explained because a better prediction is used. The distributions obtained on the model remeshed by the MAPS algorithm ( $(\mathrm{g})$ and (h) models of Fig. 4) are less significant. The same remarks have been noticed on the rabbit model.

The model (k) of Fig. 4 and (g) of Fig. 5 emphasize that the polar angle measure computed by the Butterfly scheme on the Normal mesh is not significant for our purposes. It can be explained because this latter remeshing algorithm uses a Butterfly subdivision scheme to construct the semi-regular mesh having its high-frequency details concentrated in the surface normal.

Taking into account the direction of the wavelet coefficients relating to their associated surface normal produces a distribution which better detects the high curvatures and differentiate the regions having a close roughness measure than with the other quantities. This observation is totally appropriate for segmenting the rabbit model for which the coefficients norm distribution is not as significant as for the Venus one. Moreover, for these two considered ranges, the distribution appears more significant on the Normal mesh. Consequently, in order to benefit from the best distributions for all of our roughness measures, we have considered the midpoint analysis on the Normal meshes for the following steps of our algorithm.

Finally, the various butterfly schemes, presented in sect. 3.2 have given comparable results for all the considered quantities, hence we have presented the results for the simpler one (classical scheme using the same stencil for regular and extraorinary vertices).

\subsection{Mesh classification and segmentation results}

The classification and segmentation results can't be computed directly on the semi-regular finer model, in order to benefit from the wavelet coefficients norm and polar angle. But it's easy to project the measure obtained on the finer mesh for our adaptive compression purpose. The models (m-p) of Fig. 4 and (i-l) of Fig. 5 illustrate the classification and segmentation steps of our algorithm. As explained previously in sect. 3.3.2, the 2-clusters classification have given the best results for the two models.

On the rabbit model, the classification is more difficult because the mesh have globally a more constant roughness than on Venus model. To obtain a good segmentation we then have mainly based the classification on the polar angle distribution which better differentiate the regions than with the norm measure. Moreover the consideration of the polar angle quantities (in addition to the norm) have produced a better segmentation of the 
Venus hair. The colors used for separating the connex regions were randomly generated and are not associated with a special value.

We can see that the high frequencies are globally well partitioned, but some of them, appearing in the initial mesh can't be considered. In order to obtain a better segmentation we could use a propagation of the roughness information in the coarser levels. We are actually working on a graph construction which could help us to propagate this information in all the resolution levels.

\section{CONCLUSION AND FUTURE WORK}

We have presented a new MR analysis for mesh segmentation in regions having the same surface properties in term of roughness. The main contribution of this work is the use of the lifting scheme to build a hierarchy of segmentations, each one of them keeping the global appearance of the object at different levels of detail. Our framework is able to treat any kind of semi-regular mesh and its originality comes from using the wavelet coefficients to reflect the meshes surface rougness.

The next step in this work is to use the resulting hierarchy of segmentations for building a local wavelet analysis which could serve for adaptive compression, visualisation, smoothing, denoising or watermarking.

The presented results show that the wavelet coefficients norm and polar angle are relevant measures to identify the roughness of the surface for the two analysed models. But the results could be improved by considering the variance and the histogram distribution of the various measures in order to better counterbalance them. This latter remark could also serve to automatically process the region merging threshold which remains a user defined parameter.

Finally, the boundaries of the segmented regions we have obtained can be cleaned by using the boundary rectification of Lavoue et al. ${ }^{18}$ But as this algorithm is more adapted for CAD objects, the Katz and Tal ${ }^{29}$ fuzzy decomposition, based on the geodesic distance could be adapted to give better results for natural objects.

\section{ACKNOWLEDGMENTS}

This work is supported by France Télécom R\&D Rennes within the framework of the CoSurf project.

\section{REFERENCES}

1. M. Lounsbery and T. D. DeRose and J. Warren, "Multiresolution Analysis for Surfaces of Arbitrary Topological Type," ACM Transactions on Graphics 16(1), pp. 34-73, 1997.

2. M. Eck, T. DeRose, T. Duchamp, H. Hoppe, M. Lounsbery, and W. Stuetzle, "Multiresolution analysis of arbitrary meshes," in SIGGRAPH'95: Proceedings of the 22nd annual conference on Computer graphics and interactive techniques, pp. 173-182, (New York, NY, USA), 1995.

3. P. Gioia, "Reducing the number of wavelet coefficients by geometric partitioning," Comput. Geom. Theory Appl. 14(1-3), pp. 25-48, 1999.

4. A. W. F. Lee, W. Sweldens, P. Schröder, L. Cowsar, and D. Dobkin, "MAPS: Multiresolution Adaptive Parameterization of Surfaces," Computer Graphics 32(Annual Conference Series), pp. 95-104, 1998.

5. I. Guskov, K. Vidimce, W. Sweldens, and P. Schröder, "Normal Meshes," in SIGGRAPH 2000, Computer Graphics Proceedings, pp. 95-102, 2000.

6. C. T. Loop, "Smooth subdivision surfaces based on triangles," Master's thesis, Department of Mathematics, University of Utah, Salt Lake City, 1987.

7. N. Dyn, D. Levin, and J. A. Gregory, "A butterfly subdivision scheme for surface interpolation with tension control," ACM Trans. Graph. 9(2), pp. 160-169, 1990.

8. A. Khodakovsky and I. Guskov, "Compression of Normal Meshes," in Geometric Modeling for Scientific Visualization. Springer-Verlag, 2003.

9. F. Payan and M. Antonini, "Mean square error approximation for wavelet-based semiregular mesh compression," Transactions on Visualization and Computer Graphics (TVCG) 12, July/August 2006.

10. M. Bertram, "Biorthogonal loop-subdivision wavelets," Computing 72(1-2), pp. 29-39, 2004. 
11. D. Li, K. Qin, and H. Sun, "Unlifted loop subdivision wavelets," in PG'04: Proceedings of the Computer Graphics and Applications, 12th Pacific Conference on (PG'04), pp. 25-33, IEEE Computer Society, (Washington, DC, USA), 2004.

12. A. Khodakovsky, P. Schröder, and W. Sweldens, "Progressive Geometry Compression," in SIGGRAPH 2000, Computer Graphics Proceedings, pp. 271-278, 2000.

13. M. Garland, A. Willmott, and P. S. Heckbert, "Hierarchical face clustering on polygonal surfaces," in SI3D '01: Proceedings of the 2001 symposium on Interactive 3D graphics, pp. 49-58, ACM Press, (New York, NY, USA), 2001.

14. D. Cohen-Steiner, P. Alliez, and M. Desbrun, "Variational shape approximation," ACM Trans. Graph. 23(3), pp. 905-914, 2004.

15. A. P. Mangan and R. T. Whitaker, "Partitioning 3D Surface Meshes Using Watershed Segmentation," IEEE Transactions on Visualization and Computer Graphics 5, pp. 308-321, October/December 1999.

16. Y. Sun, D. Page, and J. Paik, "Triangle Mesh-Based Edge Detection And Its Application To Surface Segmentation And Adaptive Surface Smoothing," in IEEE International Conference on Image Processing, 3, pp. 825-28, (Rochester, NY, USA), 2002.

17. A. Razdan and M. Bae, "A hybrid approach to feature segmentation of triangle meshes," Computer-Aided Design 35(9), pp. 783-789, 2003.

18. G. Lavoué, F. Dupont, and A. Baskurt, "Curvature tensor based triangle mesh segmentation with boundary rectification," in Computer Graphics International, pp. 10-17, 2004.

19. A. Gersho and R. M. Gray, Vector quantization and signal compression, Kluwer Academic Publishers, Norwell, MA, USA, 1991.

20. S. Mallat, "A theory for multiresolution signal decomposition: The wavelet representation," IEEE Trans. Pat. Anal. Mach. Intell. 11, pp. 674-693, 1989.

21. W. Sweldens, "The lifting scheme: A custom-design construction of biorthogonal wavelets," Appl. Comput. Harmon. Anal. 3(2), pp. 186-200, 1996.

22. D. Zorin, P. Schröder, and W. Sweldens, "Interpolating subdivision for meshes with arbitrary topology," Computer Graphics 30(Annual Conference Series), pp. 189-192, 1996.

23. A. Certain, J. Popovic, T. DeRose, T. Duchamp, D. Salesin, and W. Stuetzle, "Interactive multiresolution surface viewing," in SIGGRAPH'96: Proceedings of the 23rd annual conference on Computer graphics and interactive techniques, pp. 91-98, ACM Press, (New York, NY, USA), 1996.

24. D. Cohen-Steiner and J.-M. Morvan, "Restricted delaunay triangulations and normal cycle," in $S C G$ '03: Proceedings of the nineteenth annual symposium on Computational geometry, pp. 312-321, ACM Press, (New York, NY, USA), 2003.

25. G. Taubin, "Estimating the tensor of curvature of a surface from a polyhedral approximation," in ICCV'95: Proceedings of the Fifth International Conference on Computer Vision, p. 902, IEEE Computer Society, (Washington, DC, USA), 1995.

26. M. Meyer, M. Desbrun, P. Schröder, and A. H. Barr, "Discrete Differential-Geometry Operators for Triangulated 2-Manifolds," in Visualization and Mathematics III, H.-C. Hege and K. Polthier, eds., pp. 35-57, Springer-Verlag, Heidelberg, 2003.

27. G. Lavoué, E. D. Gelasca, F. Dupont, A. Baskurt, and T. Ebrahimi, "Perceptually driven 3D distance metrics with application to watermarking," in SPIE Applications of Digital Image Processing XXIX, (San Diego), Aug. 2006.

28. http://www.cgal.org

29. S. Katz and A. Tal, "Hierarchical mesh decomposition using fuzzy clustering and cuts," ACM Trans. Graph. 22(3), pp. 954-961, 2003. 


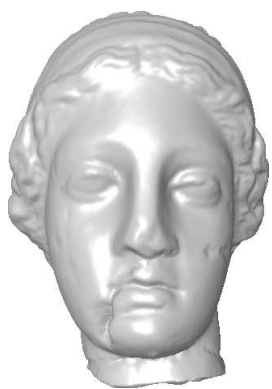

(a) Original semi-regular mesh (327 680 faces)

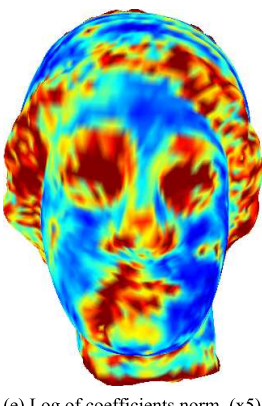

(e) Log of coefficients norm (x5)
(midpoint analysis - Normal mesh)

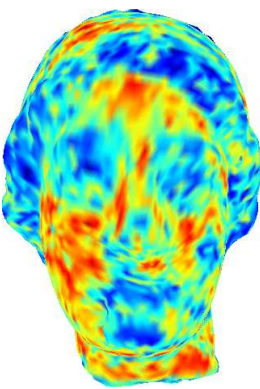

(i) Log of coefficients polar angle midpoint analysis - Normal mes

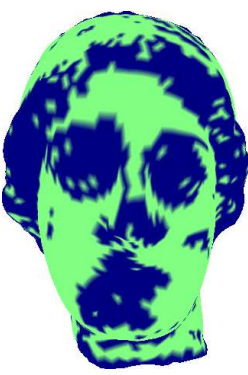

(m) K-Means algorithm based on the log of coefficients norm

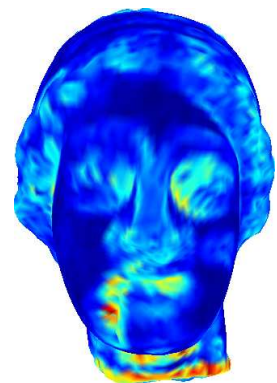

(b) Roughness information on level 2 (20 480 faces)
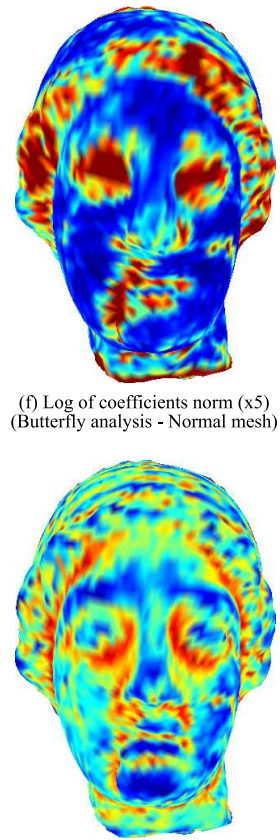

(j) Log of coefficients polar angle midpoint analysis - Normal mes

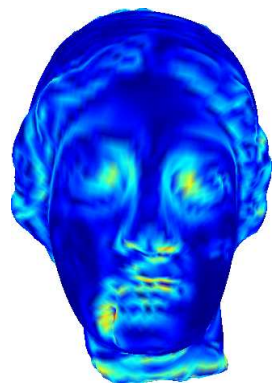

(c) Normal variation between the othed version

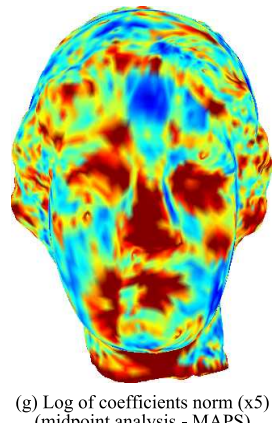

(midpoint analysis - MAPS)

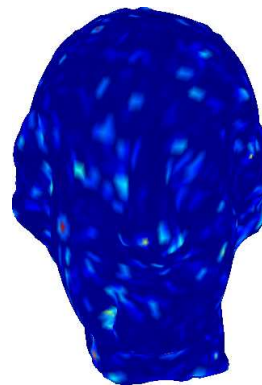

(k) Log of coefficients polar angl Butterfly analysis - Normal mesh

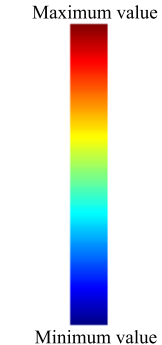

(d) Color scale used

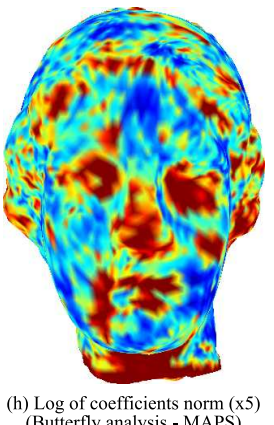

(Butterfly analysis - MAPS)

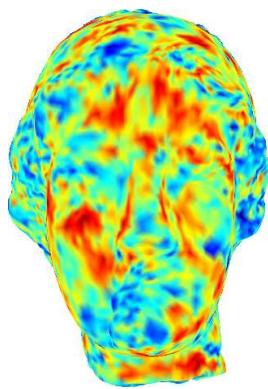

(1) Log of coefficients polar angle midpoint analysis - MAPS

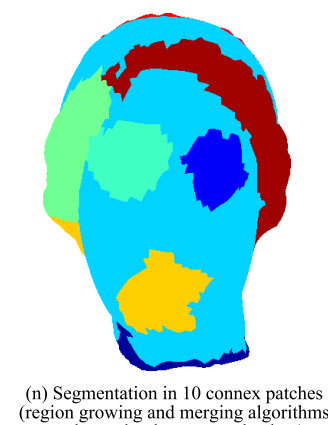

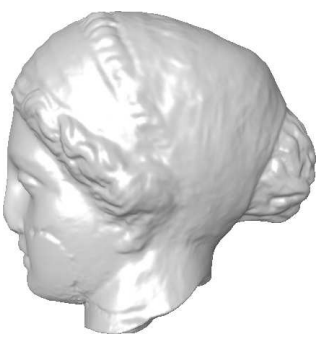

(o) Original semi-regular mes (327 680 faces)

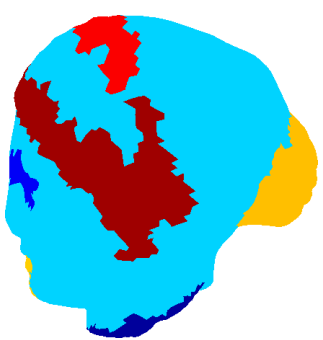

(p) Segmentation in 10 connex patches used - rondomly genented colors)

Figure 4. (b,c) Distribution of the surface roughness computed with two different algorithms on the $2^{\text {nd }}$ resolution level of the Venus model ; (d) Color scale used for (b,c) and (e-l) models ; (e-h) Distribution of the logarithm of the wavelet coefficients norm on the $2^{\text {nd }}$ resolution level, for various prediction schemes, using two different remeshing algorithms (the values have been increased by 5 to stretch the distribution, originally concentrated near the origin) ; (i-l) Distribution of the logarithm of the wavelet coefficients polar angle on the $2^{\text {nd }}$ resolution level $;(\mathrm{m})$ Classification of the $2^{\text {nd }}$ resolution level into two clusters based on the wavelet coefficients norm and polar angle (midpoint analysis on a Normal Mesh) ; $(\mathrm{n}, \mathrm{p})$ Resulting 10 connex patches obtained by the region growing and merging algorithms. 


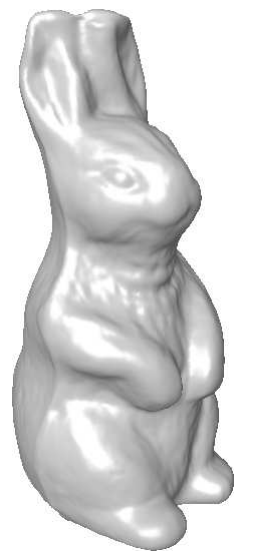
(a) Original semi-regular mesh
(141 312 faces)

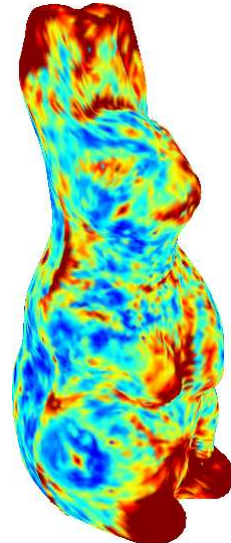

(e) Log of coefficients norm (x5) (e) Loint analysis - Normal mesh)
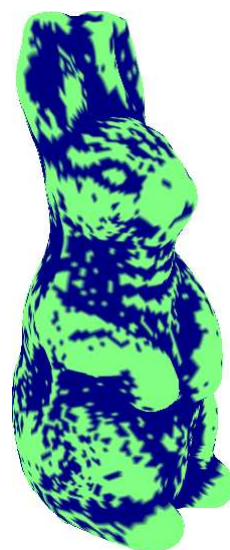

(i) K-Means algorithm mainly polar angle ( 2 clusters)
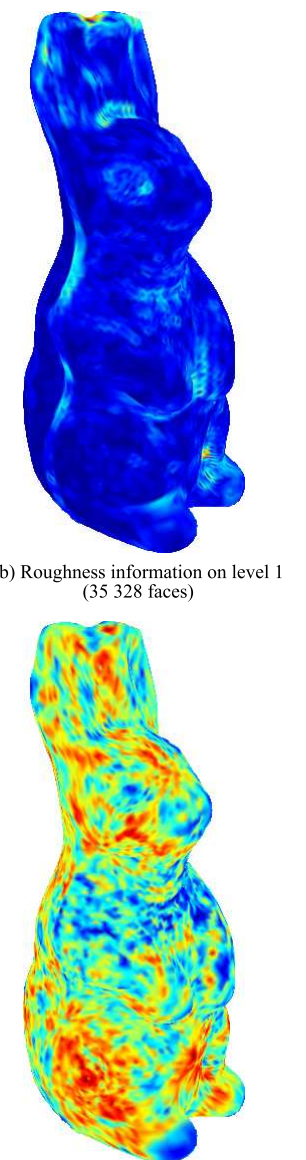

(f) Log of coefficients polar angle midpoint analysis - Normal mesh

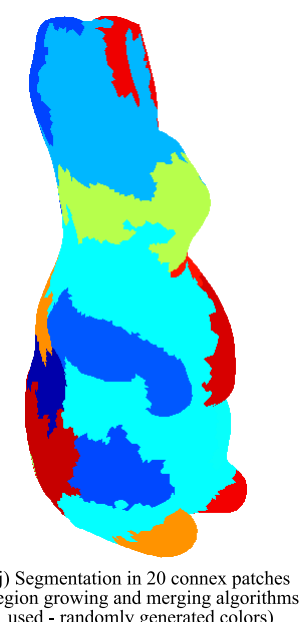

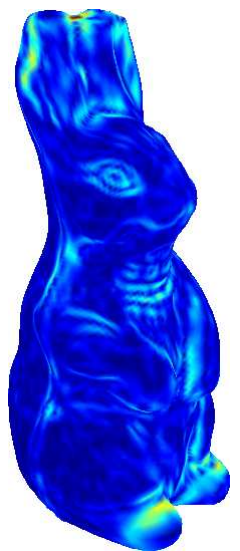

(c) Normal variation between the

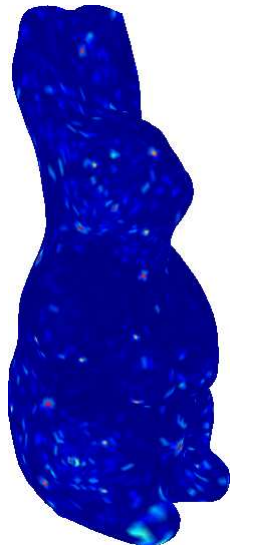

(g) Log of coefficients polar angle (ranging between 0 and $90^{\circ}$ )
Butterfly analysis - Normal mesh

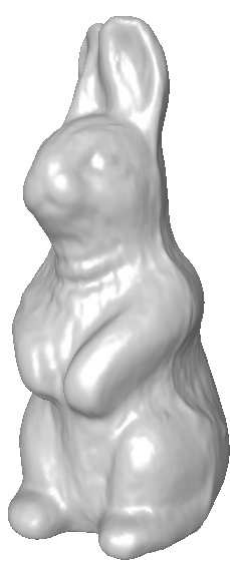

(k) Original semi-regular mesh (141 312 faces)

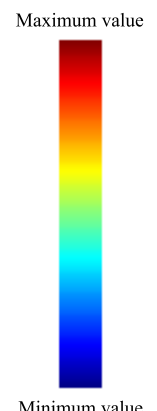

(d) Color scale used

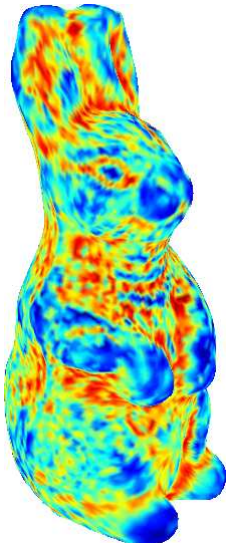

(h) Log of coefficients polar angl (ranging between 0 and $180^{\circ}$ )

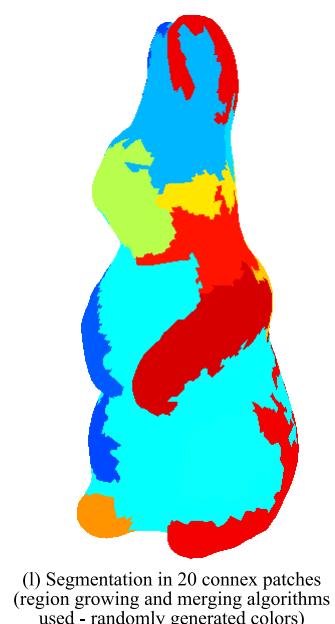

Figure 5. (b,c) Distribution of the surface roughness computed with two different algorithms on the $1^{\text {st }}$ resolution level of the rabbit model ; (d) Color scale used for (b,c) and (e-h) models ; (e-h) Distribution of the logarithm of the wavelet coefficients norm and polar angle on the $1^{\text {st }}$ resolution level (midpoint and butterfly analysis on a Normal Mesh). The norm has been increased by 5 to stretch the distribution, originally concentrated near the origin ; (i) Classification of the $1^{\text {st }}$ resolution level into two clusters mainly based on the wavelet coefficients polar angle distribution (midpoint analysis on a Normal Mesh $) ;(\mathrm{j}, \mathrm{l})$ Resulting 20 connex patches obtained by the region growing and merging algorithms. 